The incidence rate of atherosclerotic CVE was $16.3 / 1000$ patient-years (95\% Cl: 8.8-30.3), twice as high as expected by QRISK2. Analysis showed statistical difference with males $(R R=6.49, p=0.002)$ and white patients $(R R=5.04$, $\mathrm{p}=0.02$ ) although the statistical significance for white ethnicity was lost after adjusting for age and gender. Statistical analysis showed association between disease duration and higher incidence of atherosclerotic CVE, with a rate reduction of 0.7 per year from disease onset $(p=0.01)$ after adjusting for age and gender.

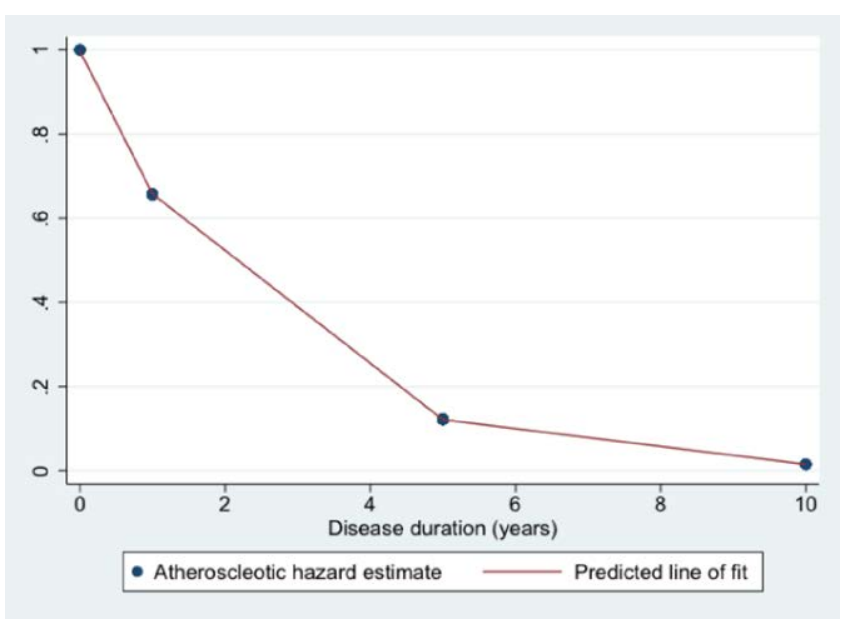

Fig. 1. Atherosclerotic risk by disease duration

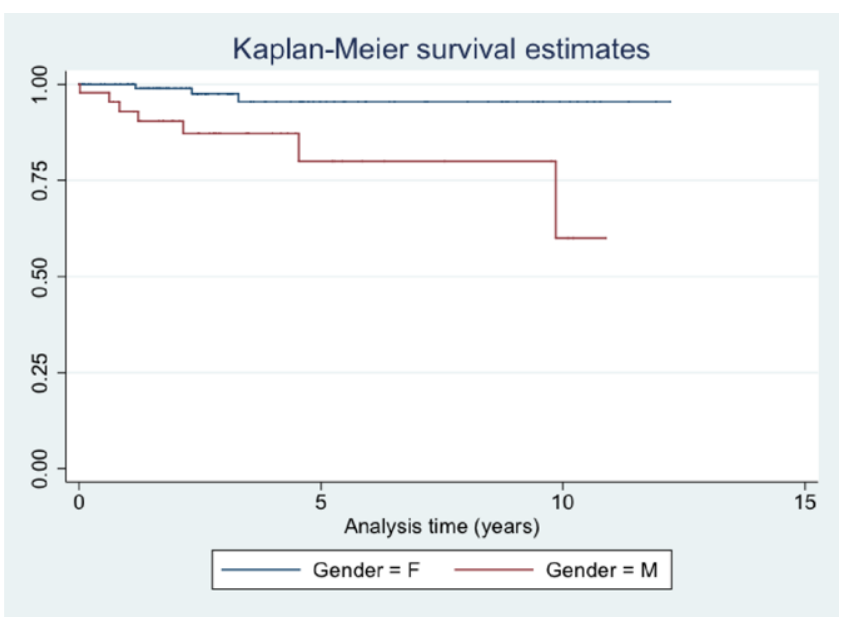

Fig. 2. Kaplan-Meier survival plot to atherosclerotic event

Conclusion: Our results suggest that atherosclerotic cardiovascular risk in inflammatory myopathies is underestimated, particularly early on the disease. Based on our results we would recommend a low threshold for primary prevention of atherosclerosis including the use of statins particularly for the first year after diagnosis.

Disclosure of Interests: None declared

DOI: 10.1136/annrheumdis-2020-eular.4926

\section{THU0336 IMMUNE CHECKPOINT INHIBITOR-RELATED MYOSITIS: A RETROSPECTIVE COHORT STUDY}

J. Aldrich ${ }^{1}$, X. Pundole ${ }^{1}$, S. Tummala ${ }^{1}$, C. Andersen ${ }^{1}$, N. Abdel-Wahab ${ }^{1}$, N. Palaskas ${ }^{1}$, A. Deswal ${ }^{1}$, M. Suarez-Almazor ${ }^{1} .{ }^{1}$ The University of Texas MD Anderson Cancer Center, Houston, United States of America

Background: Myositis is a rare immune checkpoint inhibitor (ICl)-related adverse event frequently associated with myasthenia gravis (MG) and myocarditis (MC) leading to mortality rates up to $52 \%$. $^{1}$

Objectives: To characterize the presentation, course and outcomes of patients with ICl-related myositis alone or with overlap syndrome (myositis with MG or $\mathrm{MC}$ or both).

Methods: We retrospectively identified a cohort of patients treated with $\mathrm{ICl}$ at MD Anderson Cancer Center between 2016 and 2019. Suspected myositis was identified using International Classification of Disease version 10 codes and confirmed by electronic medical record review of muscle enzymes, pathology, and other tests, when available. Patients with myositis alone or with overlap syndrome were compared using Fischer's exact tests and t tests.

Results: During the study period 8,636 patients received $\mathrm{ICI}$, of which 31 $(0.36 \%)$ were diagnosed with myositis: $14(45 \%)$ with myositis alone and 17 (55\%) with overlap (MG in 5, MC in 4, MG and MC in 8). Twenty patients received programmed death-1 (PD-1) or programmed death-ligand-1 (PDL-1) inhibitors, and 10 received combination PD-1/PDL-1 inhibitor with a cytotoxic T-lymphocyte associated antigen 4 (CTLA-4) inhibitor. One patient received single agent CTLA-4 inhibitor (excluded from pooled data). For the entire cohort the median age at diagnosis was 69 years (range: 40-95 years); the most common presenting symptoms were fatigue in $27(90 \%)$ patients, weakness in $24(80 \%)$, and myalgia in $23(77 \%)$; median CK was 2,236 U/L (range: $23-19,794 \mathrm{U} / \mathrm{L})$. For treatment, 22 of $30(73 \%)$ patients received at least one therapy in addition to steroids: plasmapheresis in $15(50 \%)$ patients, intravenous immune globulin (IVIG) in $12(40 \%)$, biologics in $9(30 \%)$ (rituximab in 6 , inflix imab in 5, tocilizumab in 3), tacrolimus in $6(20 \%)$, and mycophenolate mofetil in $4(13 \%)$. Median length of exposure to steroids was 47 days (range: 1-250 days). Five (17\%) patients were rechallenged with $\mathrm{ICl}$ after myositis resolution (3 with myositis alone, 2 with overlap), of which 1 (20\%) patient experienced a myositis flare. Twenty-five (83\%) patients were not rechallenged on $\mathrm{ICI}$ and 3 $(12 \%)$ of those patients had a flare. Differences between patients with myositis alone compared to those with overlap are shown in Table 1. Patients with overlap more often received a second therapy, specifically plasmapheresis and IVIG, had longer hospitalizations and greater symptom burden at discharge. Overall death between groups was similar; however death attributed to the adverse event occurred only in those with overlap.

Table 1. Myositis alone vs. Overlap

\begin{tabular}{|c|c|c|c|}
\hline & Myositis alone $(\mathrm{N}=13)$ & $\begin{array}{l}\text { Overlap } \\
\qquad(\mathrm{N}=17)\end{array}$ & $P$ value \\
\hline & \multicolumn{2}{|c|}{$\mathrm{N}(\%) /$ median days [range] } & \\
\hline Time to symptom onset & $42[10-161]$ & 22 [9-149] & 0.234 \\
\hline Initial steroid dose (mg/kg day) & 1.7 & 1.8 & 0.187 \\
\hline Second therapy & $7(54)$ & $15(88)$ & 0.049 \\
\hline Plasmapheresis & $3(23)$ & $12(71)$ & 0.025 \\
\hline IVIG & $1(8)$ & $11(65)$ & 0.002 \\
\hline \multicolumn{4}{|l|}{ Outcomes } \\
\hline Hospitalization length & 5 [2-50] & $24[7-92]$ & 0.019 \\
\hline Respiratory failure & $0(0)$ & $13(76)$ & \\
\hline Symptoms at discharge & & & 0.047 \\
\hline Improved & $8(62)$ & $6(35)$ & \\
\hline Resolved & $3(23)$ & $1(6)$ & \\
\hline \multicolumn{4}{|l|}{ Death } \\
\hline Overall & $8(62)$ & $12(71)$ & 0.706 \\
\hline Adverse event & $0(0)$ & $7(41)$ & \\
\hline
\end{tabular}

Conclusion: Our results represent the largest cohort of $\mathrm{ICl}$-related myositis to date. Patients with overlap syndrome are treated more aggressively and have worse outcomes than those with myositis alone. Prospective studies are warranted to determine risk factors for developing myositis or overlap syndrome and to determine optimal treatment.

References:

[1] Anquetil BC, Salem LJ-E, Lebrun-Vignes JB, et al. Immune Checkpoint Inhibitor-Associated Myositis: Expanding the Spectrum of Cardiac Complications of the Immunotherapy Revolution. Circulation. 2018;138(7): 743-745.

Disclosure of Interests: None declared

DOI: 10.1136/annrheumdis-2020-eular.605

\section{THU0337 \\ THE EFFECTIVENESS OF PLASMA EXCHANGE THERAPY FOR ANTI-MDA5 ANTIBODY-POSITIVE REFRACTORY INTERSTITIAL LUNG DISEASE}

$\underline{\text { Y. Abe }}{ }^{1}$, T. Kuga ${ }^{1}$, M. Kusaoi ${ }^{1}$, K. Tada ${ }^{1}$, K. Yamaji ${ }^{1}$, N. Tamura ${ }^{1}$. 'Juntendo University, Internal Medicine and Rheumatology, Bunkyo City, Japan

Background: This is an extended report of our study [1]. Anti-melanoma differentiation-associated gene 5 (MDA5) antibodies, which are closely related to interstitial lung disease (ILD) with or without rapid progression, are a type of myositis-specific autoantibody. Since rapid progressive-ILD (RP-ILD) with antiMDA5 antibodies is refractory and fatal, intensive immunosuppressive therapy with combination calcineurin inhibitor, and intravenous pulse cyclophosphamide was developed, and was shown to improve patient survival and prognosis [2]. However, $20-30 \%$ of cases were still fatal, and several additiona 